\title{
New Probability Distributions in Astrophysics: IV. The Relativistic Maxwell-Boltzmann Distribution
}

\author{
Lorenzo Zaninetti \\ Physics Department, via P.Giuria 1, Turin, Italy \\ Email: zaninetti@ph.unito.it
}

How to cite this paper: Zaninetti, L. (2020) New Probability Distributions in Astrophysics: IV. The Relativistic Maxwell-Boltzmann Distribution. International Journal of Astronomy and Astrophysics, 10, 302-318. https://doi.org/10.4236/ijaa.2020.104016

Received: October 15, 2020

Accepted: December 7, 2020

Published: December 10, 2020

Copyright $\odot 2020$ by author(s) and Scientific Research Publishing Inc. This work is licensed under the Creative Commons Attribution International License (CC BY 4.0).

http://creativecommons.org/licenses/by/4.0/

\begin{abstract}
Two relativistic distributions which generalize the Maxwell Boltzman (MB) distribution are analyzed: the relativistic $\mathrm{MB}$ and the Maxwell-Jüttner (MJ) distribution. For the two distributions, we derived in terms of special functions the constant of normalization, the average value, the second moment about the origin, the variance, the mode, the asymptotic behavior, approximate expressions for the average value as function of the temperature and the connected inverted expressions for the temperature as function of the average value. Two astrophysical applications to the synchrotron emission in presence of the magnetic field and the relativistic electrons are presented.
\end{abstract}

\section{Keywords}

05.20.-y Classical Statistical Mechanics, 05.20.Dd Kinetic Theory

\section{Introduction}

The equivalent in special relativity (SR) of the Maxwell-Boltzmann (MB) distribution, see [1] [2], is the so called Maxwell-Jüttner distribution (MJ), see [3] [4]. The MJ distribution has been recently revisited. We select some approaches among others: a model for the anisotropic MJ distribution [5], an astrophysical application of the MJ distribution to the energy distribution in radio jets [6], a new family of MJ distributions characterized by the parameter $\eta$ [7] and an application to counter-streaming beams of charged particles [8]. The above approaches do not cover the determination of the statistical quantities of the MJ distribution. In this paper, the statistical parameters of the relativistic MB distribution are derived in Section 2 and those of the MJ distribution are derived in Section 3. Section 4 derives the spectral synchrotron emissivity in the framework 
of the two relativistic distributions here analyzed.

\section{The Relativistic MB Distribution}

The usual MB distribution, $f\left(v ; m, k, T_{M B}\right)$, for an ideal gas is

$$
f\left(v ; m, k, T_{M B}\right)=\frac{\sqrt{2} v^{2} \mathrm{e}^{-\frac{1}{2} \frac{v^{2} m}{k T_{M B}}}}{\sqrt{\pi}\left(\frac{k T_{M B}}{m}\right)^{\frac{3}{2}}},
$$

where $m$ is the mass of the gas molecules, $k$ is the Boltzmann constant and $T_{M B}$ is the usual thermodynamic temperature. In SR, the total energy of a particle is

$$
E=m \gamma c^{2},
$$

where $m$ is the rest mass, $c$ is the light velocity, $\gamma$ is the Lorentz factor $\frac{1}{\sqrt{1-\beta^{2}}}, \beta=\frac{v}{c}$ and $v$ is the velocity. The relativistic kinetic energy, $E_{k}$, is

$$
E_{k}=m c^{2}(\gamma-1),
$$

where the rest energy has been subtracted from the total energy, see formula (23.1) in [9]. A relativistic MB distribution can be obtained from Equation (1) replacing the classical kinetic energy $\frac{1}{2} m v^{2}$ with the relativistic kinetic energy

$$
f_{r}(v ; T)=\frac{v^{2} \mathrm{e}^{\frac{1}{T}\left(1-\frac{1}{\sqrt{1-\frac{v^{2}}{c^{2}}}}\right)}}{\int_{0}^{c} w^{2} \mathrm{e}^{\frac{1}{T}\left(1-\frac{1}{\sqrt{1-\frac{w^{2}}{c^{2}}}}\right)} \mathrm{d} w}
$$

where the relativistic temperature; $T$, is expressed in $m c^{2} / k$ units; up to now the treatment is the same of [10] at pag. 665. The above relativistic PDF nolistsep - has the velocity of the light as maximum velocity,

- becomes the usual MB distribution in the limit of low velocities,

- is not invariant for relativistic transformations.

\subsection{Variable Lorentz Factor}

We now change the variable of integration

$$
v=\frac{\sqrt{\gamma^{2}-1}}{\gamma} .
$$

The differential of the velocity, $\mathrm{d} v$,

$$
\mathrm{d} v=\frac{1}{\sqrt{\gamma^{2}-1} \gamma^{2}} \mathrm{~d} \gamma,
$$

and therefore the relativistic MB distribution in the variable $\gamma$ is 


$$
f_{r}(\gamma ; T)=\frac{32 \sqrt{\gamma^{2}-1} \mathrm{e}^{\frac{1-\gamma}{T}} T^{3}}{\gamma^{4} \mathrm{e}^{T^{-1}} G_{1,3}^{3,0}\left(\left.\frac{1}{4 T^{2}}\right|_{-1 / 2,-1,-3 / 2} ^{1}\right)},
$$

where $G$ is the Mejier $G$-function [11] [12] [13]; Figure 1 reports the above PDF for three different temperatures.

The average value or mean, $\mu$, is

$$
\mu(T)=\frac{2 T G_{1,3}^{3,0}\left(\left.\frac{1}{4 T^{2}}\right|_{-1 / 2,-1 / 2,-1} ^{1}\right)}{G_{1,3}^{3,0}\left(\left.\frac{1}{4 T^{2}}\right|_{-1 / 2,-1,-3 / 2} ^{1}\right)}
$$

the second moment about the origin is

$$
\mu(T)^{2}=\frac{4 T^{2} G_{1,3}^{3,0}\left(1 /\left.4 T^{-2}\right|_{0,-1 / 2,-1 / 2} ^{1}\right)}{G_{1,3}^{3,0}\left(1 /\left.4 T^{-2}\right|_{-1 / 2,-1,-3 / 2} ^{1}\right)},
$$

the variance, $\sigma^{2}$ is

$$
=\frac{4 T^{2}\left(G_{1,3}^{3,0}\left(\left.\frac{1}{4 T^{2}}\right|_{-1 / 2,-1,-3 / 2} ^{1}\right) G_{1,3}^{3,0}\left(\left.\frac{1}{4 T^{2}}\right|_{0,-1 / 2,-1 / 2} ^{1}\right)-\left(G_{1,3}^{3,0}\left(\left.\frac{1}{4 T^{2}}\right|_{-1 / 2,-1 / 2,-1} ^{1}\right)\right)^{2}\right.}{\left(G_{1,3}^{3,0}\left(\left.\frac{1}{4 T^{2}}\right|_{-1 / 2,-1,-3 / 2} ^{1}\right)\right)^{2}} .
$$

The mode is the real solution of the following cubic equation in $\gamma$

$$
\gamma^{3}+3 T \gamma^{2}-\gamma-4 T=0
$$

which has the real solution

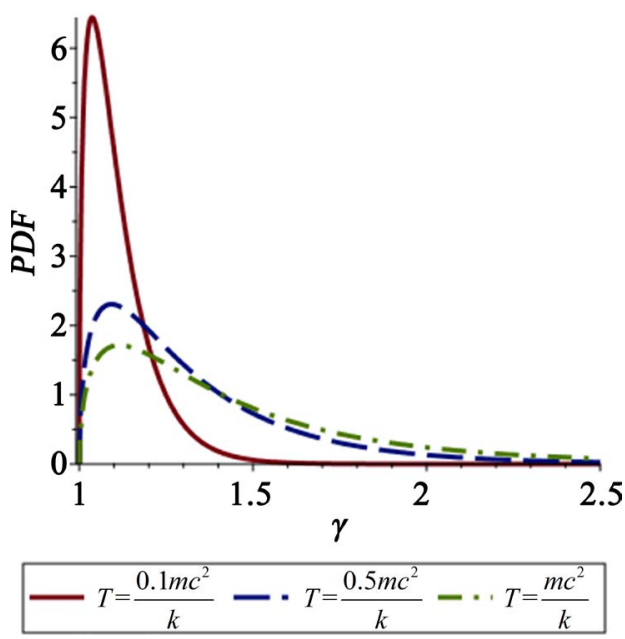

Figure 1. The PDF of the relativistic MB as function of $\gamma$ for different values of $T$. 


$$
\begin{aligned}
\text { mode }= & \frac{1}{6} \sqrt[3]{324 T-216 T^{3}+12 \sqrt{-1296 T^{4}+621 T^{2}-12}} \\
& -6 \frac{-1 / 3-T^{2}}{\sqrt[3]{324 T-216 T^{3}+12 \sqrt{-1296 T^{4}+621 T^{2}-12}}}-T .
\end{aligned}
$$

At the moment of writing a closed form for the distribution function (DF) which is

$$
F_{r}(\gamma ; T)=\int_{1}^{y} f_{r}(\gamma ; T) \mathrm{d} \gamma
$$

does not exists and we therefore present a numerical integration, see Figure 2.

The asymptotic behavior of the PDF, $f_{a}$, is

$$
\begin{aligned}
f_{a}(\gamma ; T)= & \frac{1}{G_{1,3}^{3,0}\left(1 /\left.4 T^{-2}\right|_{-1 / 2,-1,-3 / 2} ^{1}\right) 2048 \gamma^{21}} T^{3}\left(65536 \gamma^{18}-32768 \gamma^{16}\right. \\
& -8192 \gamma^{14}-4096 \gamma^{12}-2560 \gamma^{10}-1792 \gamma^{8}-1344 \gamma^{6} \\
& \left.-1056 \gamma^{4}-858 \gamma^{2}-715\right) \mathrm{e}^{-\frac{\gamma}{T}} .
\end{aligned}
$$

The integration of the above approximate PDF gives an approximate DF which has a maximum percentage error of $7 \%$ in the interval $1.1<\gamma<4$ when $T=1$. The random numbers belonging to the relativistic MB can be generated through a numerical computation of the inverse function following the algorithm outlined in Sec. 4.9.1 of [14]. The above PDF has only one parameter which can be derived approximating the average value with a Pade approximant $[2,2]$

$$
\mu(T) \approx \frac{-0.061723842+1.542917977 T+0.3269078746(T-1)^{2}}{0.1069596119+0.8930403881 T+0.1511024609(T-1)^{2}} .
$$

The above approximation in the interval $0.1 \leq T<10$ has a percent error less than $1 \%$. The inverse function allows to derive $T$ as

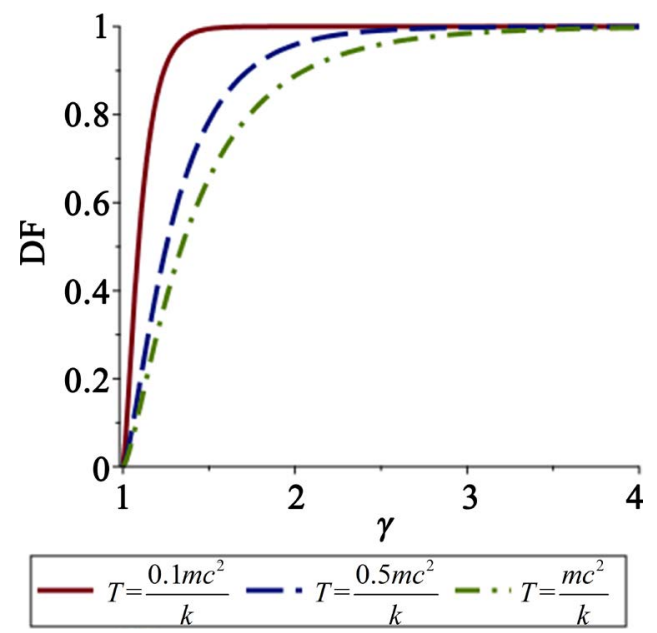

Figure 2. The numerical DF of the relativistic MB as function of $\gamma$ for different values of $T$. 


$$
T=-\frac{15.908 \times 10^{9} \bar{x}-8.89 \times 10^{9}+\sqrt{1.931 \times 10^{19} \bar{x}^{2}-5.528 \times 10^{19} \bar{x}+4.437 \times 10^{19}}}{1.511 \times 10^{9} \bar{x}-3.269 \times 10^{9}} .
$$

Here $\bar{x}$ is the sample mean defined as

$$
\bar{x}=\frac{1}{n} \sum_{i=1}^{n} x_{i}
$$

formula which is useful to derive the variance of the sample

$$
\operatorname{Var}=\frac{1}{n-1} \sum_{i=1}^{n}\left(x_{i}-\bar{x}\right)^{2}
$$

where $x_{i}$ are the n-data, see [15]. An example of random generation of points is reported in Figure 3 where we imposed $T=1$ and we found $T=1.0397$ from the generated random sample.

\subsection{Variable Velocity}

We now return to the variable velocity, the PDF is

$$
f_{r}(v ; T)=\frac{32 \sqrt{-\frac{v^{2}}{v^{2}-1}} \mathrm{e}^{\frac{\sqrt{-v^{2}+1}-1}{\sqrt{-v^{2}+1}}} \sqrt{-v^{2}+1} T^{3} v}{\mathrm{e}^{T^{-1}} G_{1,3}^{3,0}\left(1 /\left.4 T^{-2}\right|_{-1 / 2,-1,-3 / 2} ^{1}\right)},
$$

where $v$ is expressed in $c=1$ units. The mode is a solution of a sextic equation, see [16], in $V$

$$
-4 T^{2} v^{6}+12 T^{2} v^{4}-v^{4}-12 T^{2} v^{2}+4 T^{2}=0,
$$

which has the following real solution

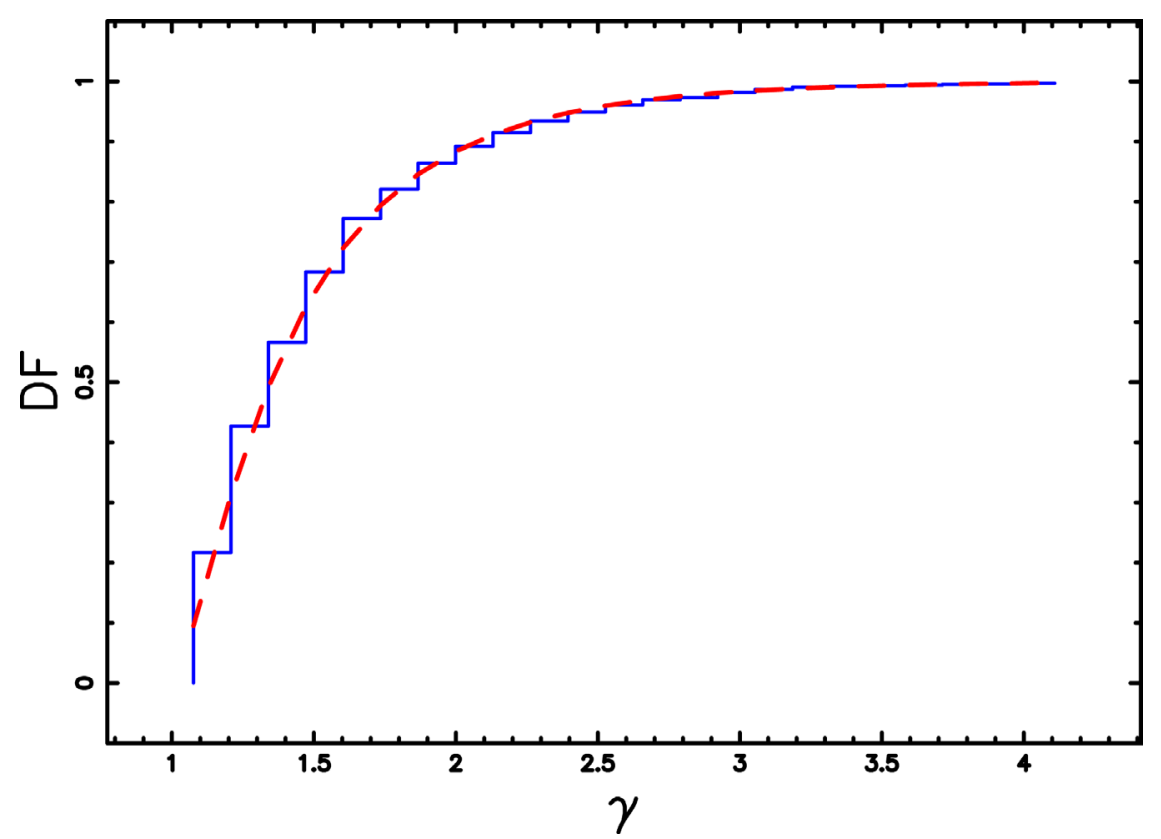

Figure 3. The DF for 3000 random points generated according to the relativistic MB (blue steps diagram) and the theoretical DF (red dashed line) see 3. 


$$
\begin{aligned}
\text { mode }= & \frac{1}{6}\left(3 \frac{\sqrt[3]{24 \sqrt{3} \sqrt{27 T^{2}-1} T^{3}-216 T^{4}+36 T^{2}-1}}{T^{2}}\right. \\
& \left.-3 \frac{24 T^{2}-1}{T^{2} \sqrt[3]{24 \sqrt{3} \sqrt{27 T^{2}-1} T^{3}-216 T^{4}+36 T^{2}-1}}+3 \frac{12 T^{2}-1}{T^{2}}\right)^{1 / 2} .
\end{aligned}
$$

The position of the mode for the PDF in $v$ is different from that one in $\gamma$, see Figure 4.

At the moment of writing the other statistical parameters cannot be presented in a closed form.

\section{The Maxwell Jüttner Distribution}

The PDF for the Maxwell Jüttner (MJ) distribution is

$$
f_{M J}(\gamma ; \Theta)=\frac{\gamma \sqrt{\gamma^{2}-1} \mathrm{e}^{-\frac{\gamma}{\Theta}}}{\Theta K_{2}\left(\frac{1}{\Theta}\right)},
$$

where $\Theta=\sqrt{\frac{k T_{M B}}{m c^{2}}}, m$ is the mass of the gas molecules, $k$ is the Boltzmann constant, $T_{M B}$ is the usual thermodynamic temperature and $K_{2}(x)$ is the Bessel function of second kind, see [3] [4] [5] [6]. Figure 5 reports the above PDF for three different values of $\Theta$ and Figure 6 displays the PDF as a 2-D contour.

The average value is

$$
\mu(\Theta)=\frac{-2 \Theta^{2} G_{1,3}^{2,1}\left(\left.\frac{1}{4 \Theta^{2}}\right|_{3 / 2,-1 / 2,-2} ^{1}\right)}{K_{2}\left(\frac{1}{\Theta}\right)}
$$

and the variance is

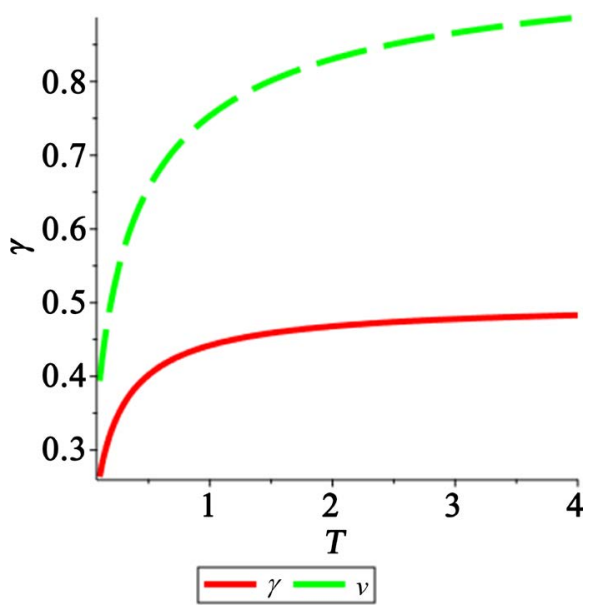

Figure 4. The position of the mode in the two PDFs: gamma variable (red full line) and $v$ variable (dashed green line). 


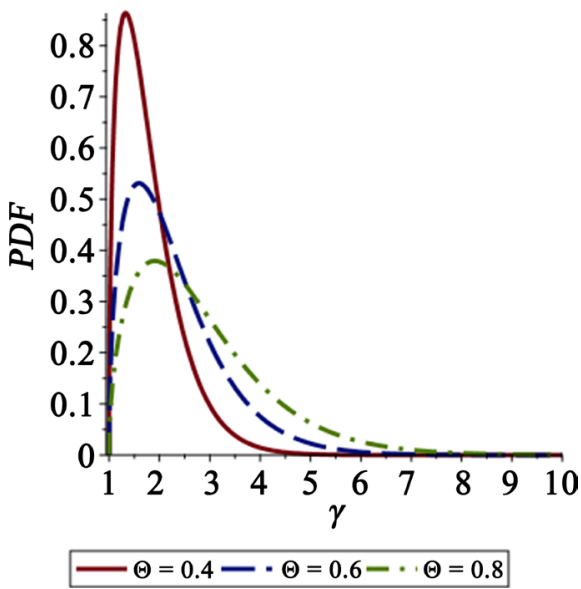

Figure 5. The PDF of the MJ as function of $\gamma$ for different values of $\Theta$.

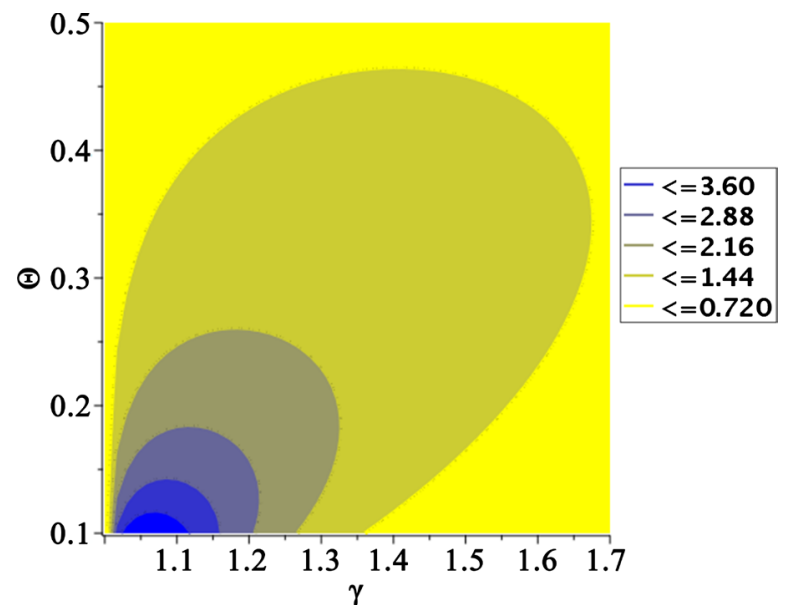

Figure 6. A 2-D contour for the MJ PDF as function of $\gamma$ and $\Theta$.

$$
\begin{aligned}
\sigma^{2}(\Theta)= & \frac{1}{\Theta^{2}\left(K_{2}\left(\frac{1}{\Theta}\right)\right)^{2}}\left(-4 \Theta^{5}\left(2 K_{1}\left(\Theta^{-1}\right) G_{1,3}^{2,1}\left(1 /\left.4 \Theta^{-2}\right|_{5 / 2,-1 / 2,-2} ^{1}\right) \Theta\right.\right. \\
& \left.\left.+\left(G_{1,3}^{2,1}\left(1 /\left.4 \Theta^{-2}\right|_{3 / 2,-1 / 2,2} ^{1}\right)\right)^{2} \Theta+K_{0}\left(\Theta^{-1}\right) G_{1,3}^{2,1}\left(1 /\left.4 \Theta^{-2}\right|_{5 / 2,-1 / 2,2} ^{1}\right)\right)\right) .
\end{aligned}
$$

The mode can be found by solving the following cubic equation

$$
\frac{\mathrm{d}}{d \gamma} f_{M J}(\gamma ; \Theta) \propto-\gamma^{3}+2 \Theta \gamma^{2}+\gamma-\Theta=0 .
$$

The real solution is

$$
\begin{aligned}
\text { mode }= & \frac{1}{6 \sqrt[3]{-36 \Theta+64 \Theta^{3}+12 \sqrt{-96 \Theta^{4}-39 \Theta^{2}-12}}} \\
& \times\left(\left(-36 \Theta+64 \Theta^{3}+12 \sqrt{-96 \Theta^{4}-39 \Theta^{2}-12}\right)^{\frac{2}{3}}\right. \\
& \left.+4 \Theta \sqrt[3]{-36 \Theta+64 \Theta^{3}+12 \sqrt{-96 \Theta^{4}-39 \Theta^{2}-12}}+16 \Theta^{2}+12\right) .
\end{aligned}
$$


The asymptotic expansion of order 10 for the PDF is

$$
f_{M J}(\gamma ; \Theta) \sim \frac{1}{\Theta K_{2}\left(\frac{1}{\Theta}\right)} \frac{\left(128 \gamma^{8}-64 \gamma^{6}-16 \gamma^{4}-8 \gamma^{2}-5\right) \mathrm{e}^{-\frac{\gamma}{\Theta}}}{128 \gamma^{6}} .
$$

The DF is evaluated with the following integral

$$
F_{M J}(\gamma ; \Theta)=\int_{1}^{\gamma} f_{M J}(\gamma ; \Theta) \mathrm{d} \gamma,
$$

which cannot be expressed in terms of special functions.

We now present some approximations for the distribution function A first approximation is given by a series expansion when, ad example, $\Theta=1$

$$
F_{M J}(\gamma ; 1)=\frac{1}{K_{2}(1)}\left(K_{2}(1)+\sqrt{\pi} \sum_{m=0}^{\infty} \frac{(-1)^{1+m} \Gamma(3-2 m, \gamma)}{\Gamma(1+m) \Gamma\left(\frac{3}{2}-m\right)}\right),
$$

which has a percent error less $<0.6 \%$ in interval $1.1<\gamma<10$ when $T=1$. A second approximation is given by an asymptotic expansion of order 50 for the PDF followed by the integration, see Figure 7. The parameter $\Theta$ can be derived from the experimental sample once the average value is modeled by a Pade approximant $[2,2]$ and the inverse function is derived

$$
\begin{aligned}
\Theta= & 0.1661 \bar{x}-0.3085+1.36051 \times 10^{-10} \\
& \times \sqrt{1.4908 \times 10^{18} \bar{x}^{2}+5.913 \times 10^{18} \bar{x}-6.5835 \times 10^{18}} .
\end{aligned}
$$

An analogous formula allows to derive $\Theta$ from the variance Var of the sample

$\Theta=\frac{1}{4} \times \frac{1.818 \times 10^{10} \mathrm{Var}+5.972 \times 10^{11}+5 \sqrt{2.277 \times 10^{20} \operatorname{Var}^{2}+7.814 \times 10^{23} \mathrm{Var}-3.597 \times 10^{22}}}{5.436 \times 10^{8} \mathrm{Var}+1.978 \times 10^{12}}$.

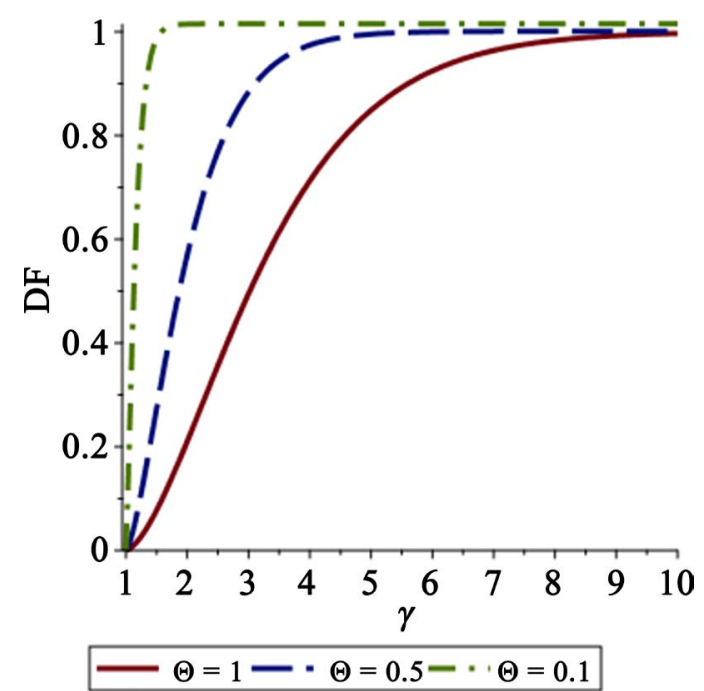

Figure 7. The numerical MJ DF as function of $\gamma$ for different values of $\Theta$. 
An example of random generation of points is reported in Figure 8 where we imposed $T=10$ and we found $T=9.97$ from formula (30) and $T=9.98$ from formula (31).

\section{Variable $\beta$}

We now change the variable of integration $\gamma$ in $\beta=\frac{v}{c}$, the PDF of the MJ is

$$
f_{M J}(\beta ; \Theta)=\frac{\sqrt{\left(1-\beta^{2}\right)^{-1}-1 \mathrm{e}^{-\frac{1}{\Theta} \frac{1}{\sqrt{1-\beta^{2}}}} \beta}}{\left(1-\beta^{2}\right)^{2} \Theta K_{2}\left(\frac{1}{\Theta}\right)},
$$

where $0 \leq \beta \leq 1$, see Figure 9. We have only one analytical result, the mode, which is found solving the following equation in $\beta$

$$
-3(\beta-1)^{3}(\beta+1)^{3}\left(\Theta\left(\beta^{2}+2 / 3\right) \sqrt{-\beta^{2}+1}-1 / 3 \beta^{2}\right) \mathrm{e}^{-\frac{1}{\sqrt{-\beta^{2}+1 \Theta}}} \beta^{2}=0 .
$$

As an example when $\Theta=0.1$ the mode is at $\beta=0.4866$ and Figure 10 reports the mode as function of $\Theta$.

The mean and the variance of the MJ distribution does not have an analytical expression and they are reported in a numerical way, see Figure 11 and Figure 12.

The DF of the $\mathrm{MJ}$ is given by the following integral

$$
F_{M J}(\beta ; \Theta)=\int_{0}^{\beta} f_{M J}(\beta ; \Theta) \mathrm{d} \beta,
$$

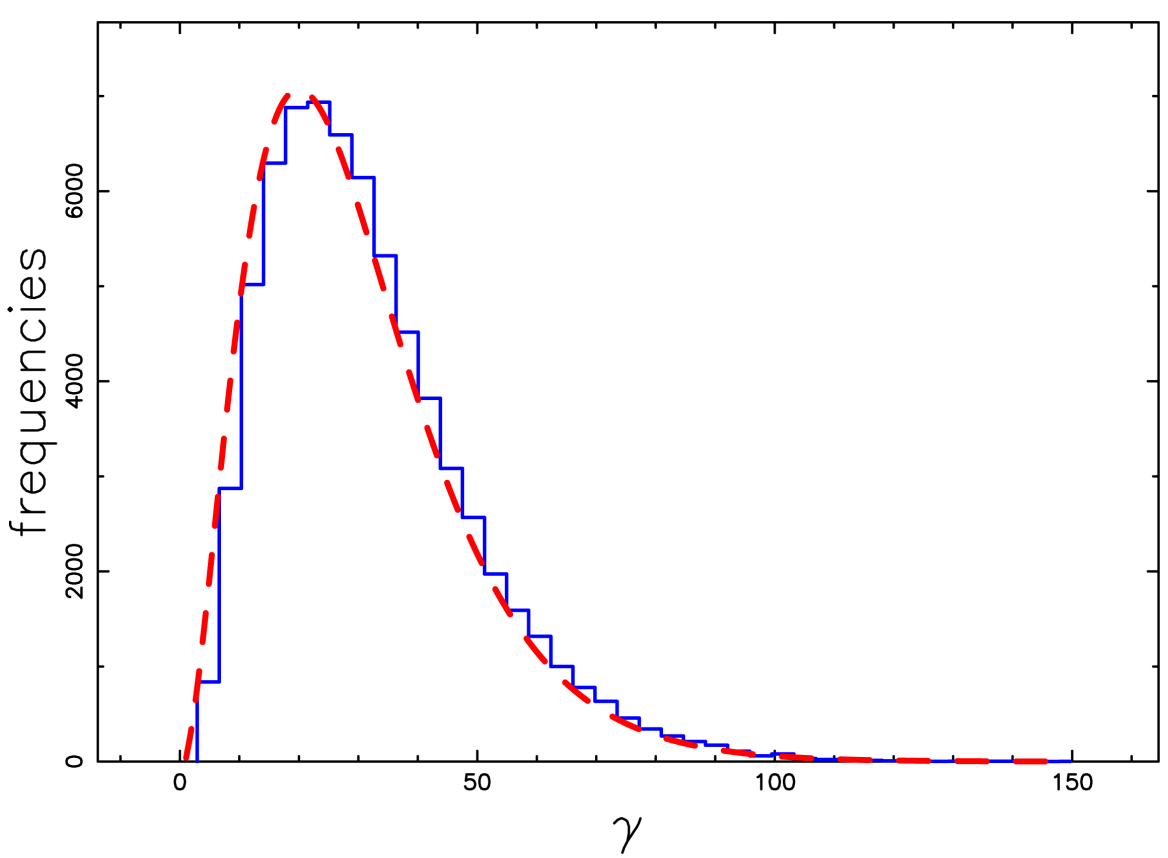

Figure 8. The PDF for 70,000 random points generated according to the MJ (blue steps) and the theoretical PDF (red dashed line), see formula (22). 


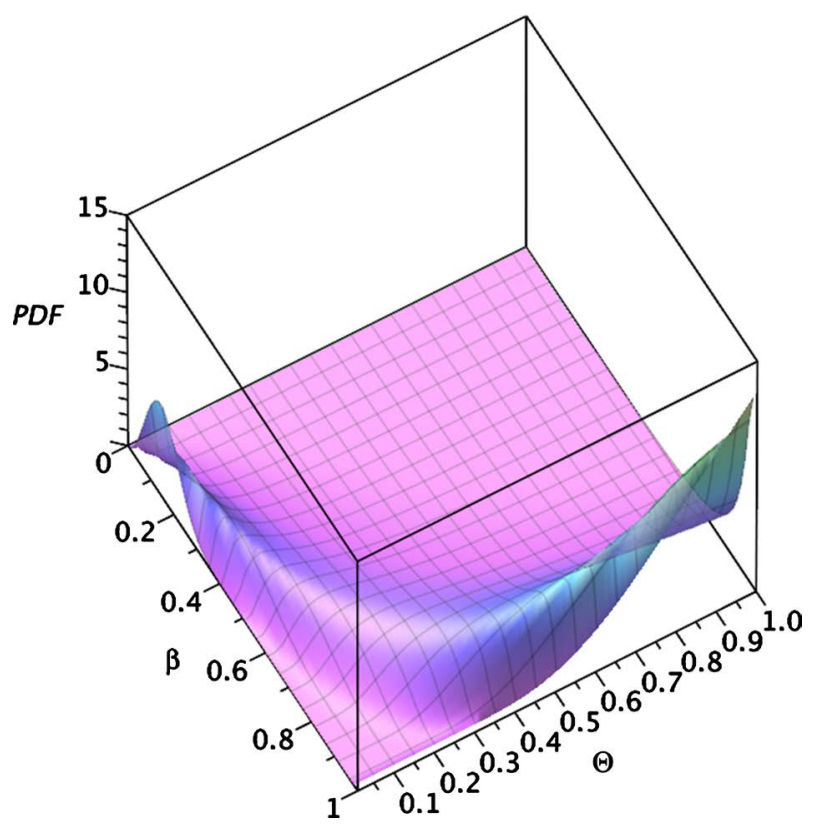

Figure 9. The 3D surface of the MJ PDF as function of $\Theta$ and $\beta$.

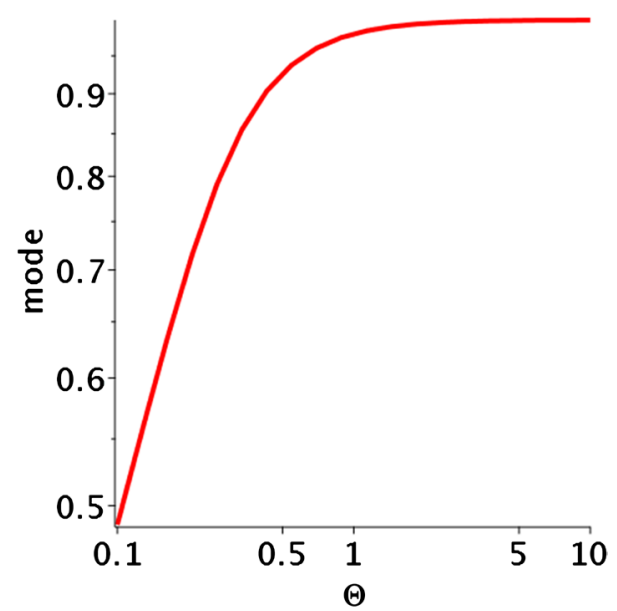

Figure 10. The mode of the MJ distribution for different values of $\Theta$.

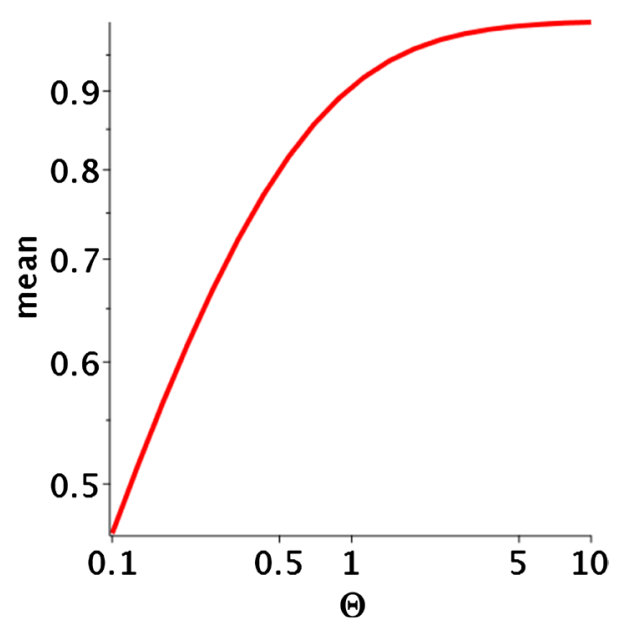

Figure 11. The mean of the MJ distribution for different values of $\Theta$. 


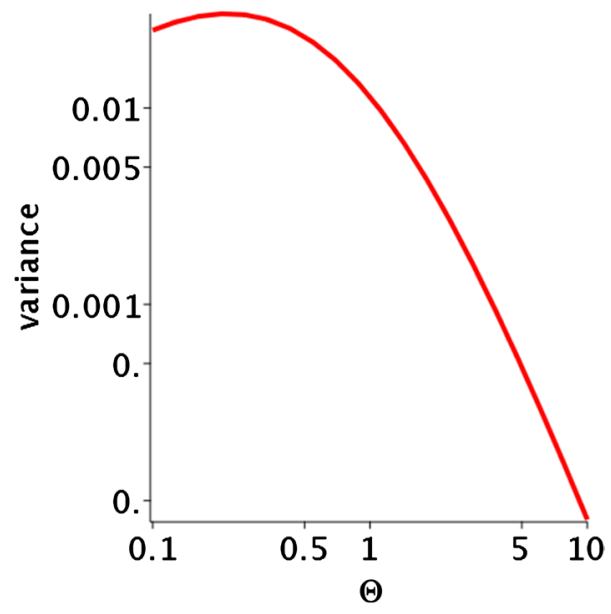

Figure 12. The variance of the MJ distribution for different values of $\Theta$.

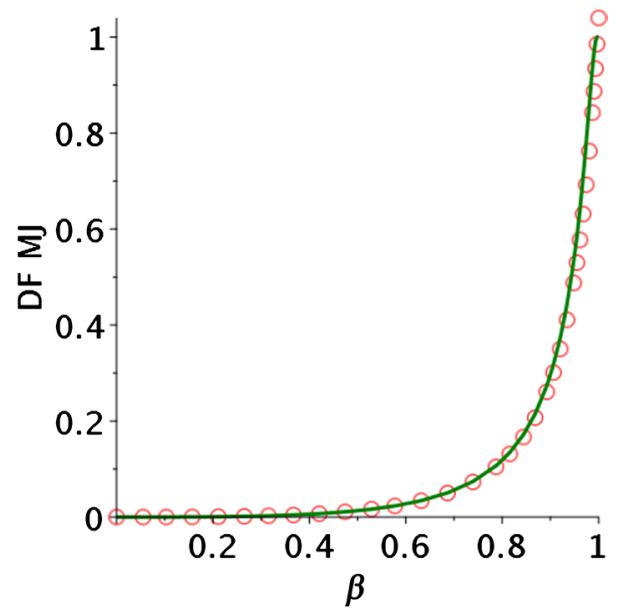

Figure 13. The numerical DF of the MJ distribution (red circles) and the Riemann approximation (green line) as function of $\beta$.

with $\beta$ in $[0,1]$ which does not have an analytical expression. An approximation is given by the Riemann sums, see [17], when $\Theta=1$

$F_{M J}(\beta ; \Theta)$

$$
=\frac{\beta \sum_{i=0}^{9} \frac{\beta}{10 K_{2}(1)} \sqrt{\left(-\frac{\beta^{2}}{100}\left(i+\frac{1}{2}\right)^{2}+1\right)^{-1}-1 \mathrm{e}^{\sqrt{-\frac{\beta^{2}}{100}\left(i+\frac{1}{2}\right)^{2}+1}}\left(i+\frac{1}{2}\right)\left(-\frac{\beta^{2}}{100}\left(i+\frac{1}{2}\right)^{2}+1\right)^{-2}}}{10},
$$

see Figure 13. The above DF has a maximum percentage error of $\approx 10 \%$ at $\beta=1$.

\section{The Astrophysical Applications}

This section reviews the synchrotron emissivity for a single relativistic electron, derives the spectral synchrotron emissivity for the two relativistic distributions here analyzed and models the observed synchrotron emission in some astro- 
physical sources.

\subsection{Synchrotron Emissivity}

The synchrotron emissivity of a single electron is

$$
\frac{\sqrt{3} e^{3} B \sin (\alpha)}{8 \pi^{2} \epsilon_{0} c m_{e}} F(x),
$$

where, according to eqn.(8.58) in [18], $e$ is the electron charge, $B$ is the magnetic field, $\alpha$ is the pitch angle, $\epsilon_{0}$ is the permittivity of free space, $c$ is the light velocity, $m_{e}$ is the electron mass, $x=$ is the ratio of the angular frequency $(\omega)$ to the critical angular frequency $\left(\omega_{c}\right)$ and

$$
F(x)=x \int_{x}^{\infty} K_{5 / 3}(z) \mathrm{d} z
$$

where $K_{5 / 3}(z)$ is the modified Bessel function of second kind with order 5/3 [13] [19]. The modified Bessel function is also known as Basset function, modified Bessel function of the third kind or Macdonald function see pag. 527 in [20]. The above function has the following analytical expression

$$
\begin{aligned}
F(x)= & -\frac{9 \sqrt{3} \sqrt[3]{2} \pi}{320 \Gamma(2 / 3)} x_{1}^{\frac{11}{3}} F_{2}\left(\frac{4}{3} ; \frac{7}{3}, \frac{8}{3} ; \frac{x^{2}}{4}\right)-\frac{x \sqrt{3} \pi}{3} \\
& +\sqrt[3]{x} 2^{\frac{2}{3}} \Gamma\left(\frac{2}{3}\right){ }_{1} F_{2}\left(-\frac{1}{3} ;-\frac{2}{3}, \frac{2}{3} ; \frac{x^{2}}{4}\right)
\end{aligned}
$$

where $1(a, b ; c ; v)$ is a regularized hypergeometric function [19] [21] [22] [13]. Figure 14 displays $F(x)$ as function of $x$.

\subsection{The Synchrotron Relativistic MB Distribution}

We start from the PDF for the relativistic MB distribution as represented by Equation (7) and we perform the following first change of variable

$$
\gamma=\frac{E}{m_{e} c^{2}},
$$

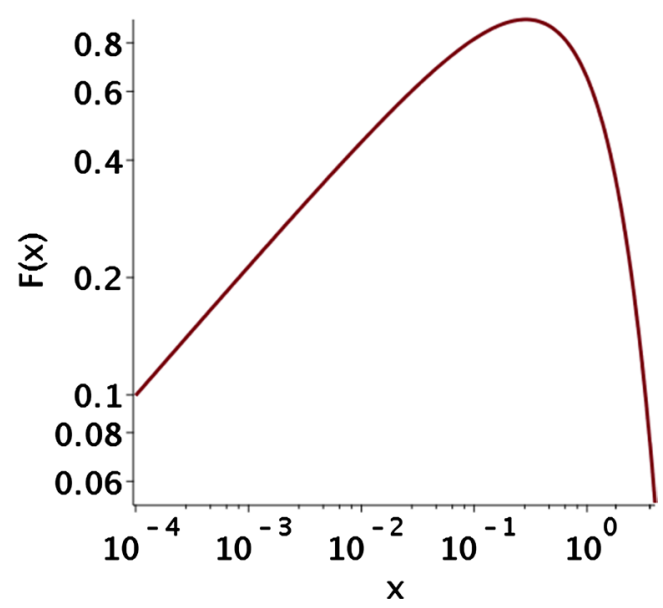

Figure 14. $F(x)$ as function of $x$ with logarithmic axes. 
where $E$ is the relativistic energy. The resulting PDF in relativistic energy is

$$
f_{r}(E ; T)=\frac{32 \sqrt{\frac{E^{2}}{m_{e}^{2} c^{4}}-1 \mathrm{e}^{\frac{1}{T}\left(1-\frac{E}{m_{e} c^{2}}\right)} T^{3} m_{e}^{3} c^{6}}}{E^{4} \mathrm{e}^{T^{-1}} G_{1,3}^{3,0}\left(\left.\frac{1}{4 T^{2}}\right|_{-\frac{1}{2},-1,-\frac{3}{2}} ^{1}\right)} .
$$

A second change of variable is

$$
E=\sqrt{\frac{v}{v_{g}}} m_{e} c^{2},
$$

produces

$$
f_{r}\left(v ; T, v_{g}\right)=\frac{16 \sqrt{\frac{v}{v_{g}}-1} \mathrm{e}^{\frac{1}{T}\left(1-\sqrt{\frac{v}{v_{g}}}\right)} T^{3} v_{g}}{v^{2} \mathrm{e}^{T^{-1}} G_{1,3}^{3,0}\left(\left.\frac{1}{4 T^{2}}\right|_{-\frac{1}{2},-1,-\frac{3}{2}} ^{1}\right) \sqrt{\frac{v}{v_{g}}}},
$$

where

$$
v_{g}=\frac{e B}{2 \pi m_{e}} .
$$

We know that $v_{g}=2.799249 \times 10^{12} B$ where $B$ is the magnetic field expressed in gauss and therefore the above PDF in frequency becomes

$$
f_{r}(v ; T, B)=\frac{7.49345 \times 10^{19} \sqrt{3.57238 \times 10^{-13} \frac{v}{B}-1 \mathrm{e}^{\frac{1}{T}}\left(1-5.97694 \times 10^{-7} \sqrt{\frac{v}{B}}\right)} T^{3} B}{v^{2} \mathrm{e}^{T^{-1}} G_{1,3}^{3,0}\left(\left.\frac{1}{4 T^{2}}\right|_{-\frac{1}{2},-1,-\frac{3}{2}} ^{1}\right) \sqrt{\frac{v}{B}} .}
$$

\subsection{The Synchrotron Maxwell Jüttner Distribution}

We start from the PDF for the Maxwell Jüttner distribution as given by Equation (22) and we perform two changes in variable as in the previous section. The resulting PDF in relativistic energy is

$$
f_{M J}(E ; \Theta)=\frac{E \sqrt{\frac{E^{2}}{m_{e}^{2} c^{4}}-1 \mathrm{e}^{-\frac{E}{m_{e} c^{2} \Theta}}}}{m_{e}^{2} c^{4} \Theta K_{2}\left(\frac{1}{\Theta}\right)} .
$$

The second PDF in $v$ is

$$
f_{M J}\left(v ; \Theta, v_{g}\right)=\frac{\sqrt{\frac{v}{v_{g}}-1 \mathrm{e}^{-\frac{1}{\Theta}} \sqrt{\frac{v}{v_{g}}}}}{2 \Theta K_{2}\left(\frac{1}{\Theta}\right) v_{g}} .
$$


The astrophysical PDF in frequency for the Maxwell Jüttner distribution is

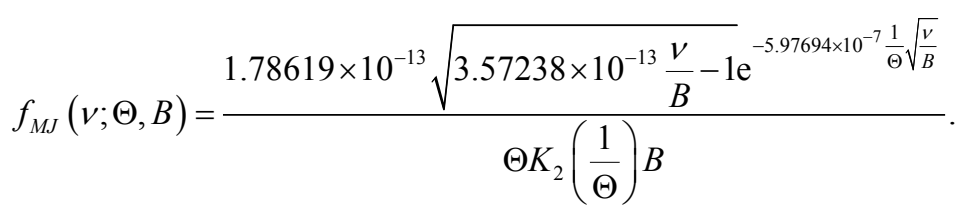

The mismatch between measured flux in Jy and theoretical flux, $S_{\text {theo }}$, can be obtained introducing a multiplicative constant $C$

$$
S_{\text {theo }}=C \times f_{M J}(v ; \Theta, B) .
$$

\subsection{The Spectrum of the Radio-Sources}

As a first example we analyze the spectrum of an extended region around M87, see as example Figure 1 in [23] where the flux in Jy as function of the frequency is reported in the range $9 \times 10^{9} \mathrm{~Hz}<v<2 \times 10^{18} \mathrm{~Hz}$. Figure 15 reports the measured and theoretical flux in the range $9 \times 10^{9} \mathrm{~Hz}<v<2 \times 10^{12} \mathrm{~Hz}$ for the quiet core of M87.

A second example is given by the radio sources with ultra steep spectra (USS) which are characterized by a spectral index, $\alpha$, lower than -1.30 when the radio flux, $S$, is proportional to $S^{\alpha}$, see [24]. As a practical example we select the cluster Abell 1914 where the measured total flux densities at $150 \mathrm{MHz}$ and 1.4 $\mathrm{GHz}$ are $S_{150}=4.68 \mathrm{Jy}$ and $S_{1.4}=34.8 \mathrm{mJy}$ which means $\alpha=-2.17$. We now evaluate the theoretical spectral index of synchrotron emission for the relativistic $\mathrm{MB}$ distribution between $150 \mathrm{MHz}$ and $1.4 \mathrm{GHz}$ when $B$ is fixed and $T$ variable, see Figure 16 and Figure 17 when $T$ and $B$ are both variables. The two

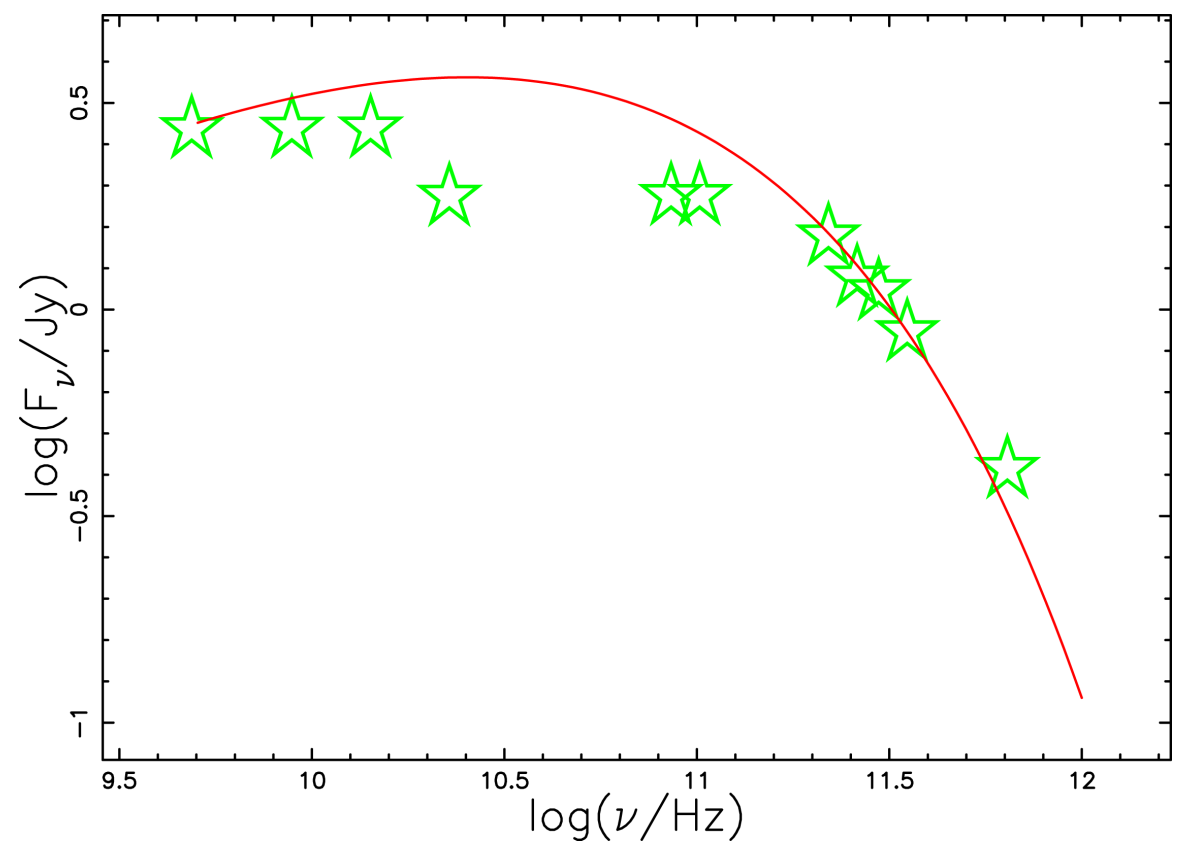

Figure 15. Measured flux in Jy (green stars) of the quiet core of M87 and the theoretical flux (red line) for the Maxwell Jüttner distribution as given by Equation (48). The numerical parameters are $B=10^{-5}$ gauss, $\Theta=30$ and $C=10^{12} \mathrm{Jy}$. 


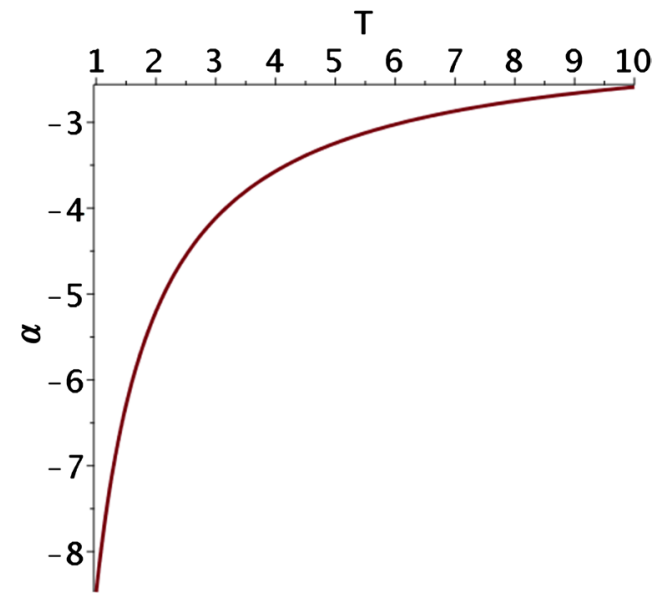

Figure 16. The spectral index of the relativistic MB as function of $T$ when $B=1.0 \times 10^{-6}$.

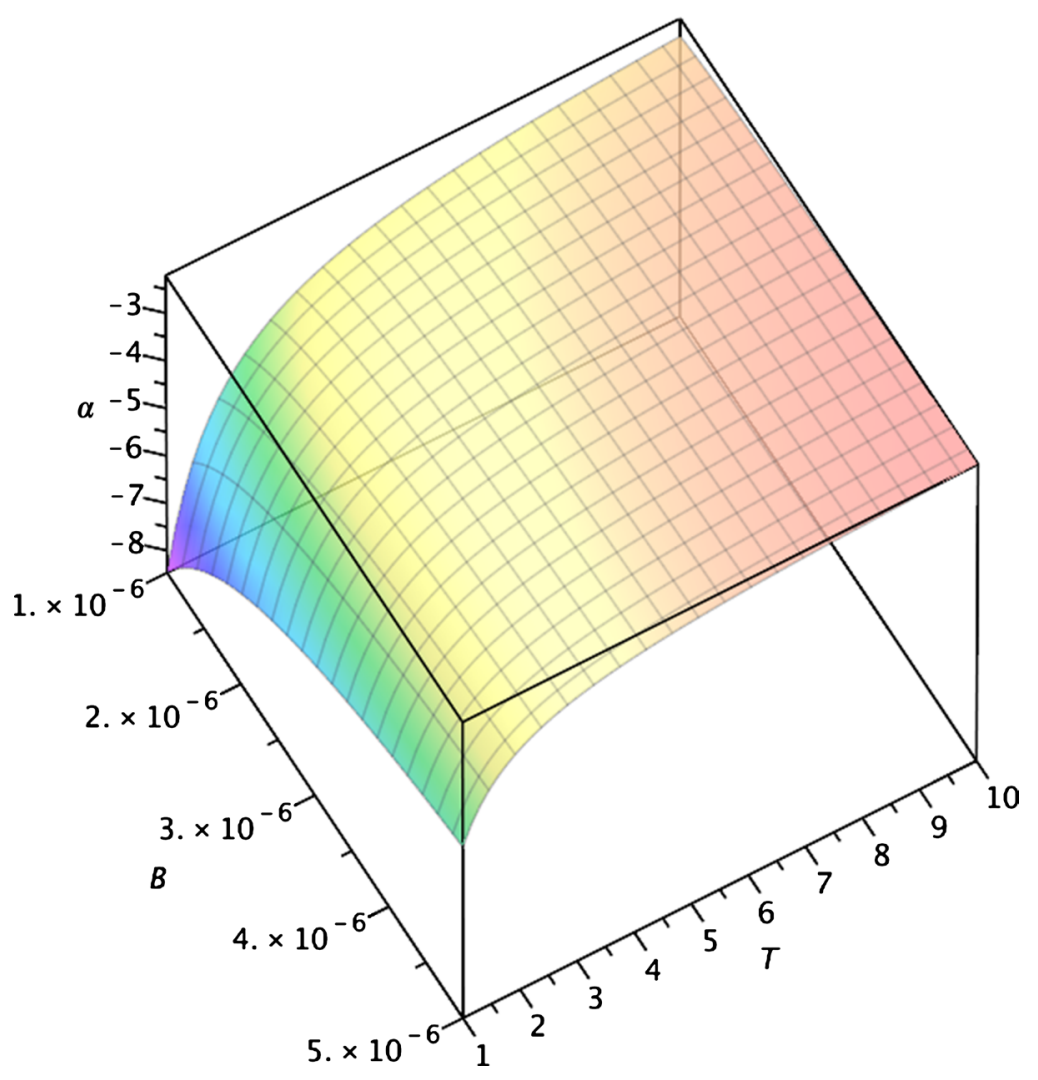

Figure 17. The spectral index of the relativistic $M B$ as function of $T$ and $B$ in gauss.

Figures above show that the theoretical spectral index is always smaller than -2 which can be considered as an asymptotic limit for high values of relativistic temperature. As an example when $B=1.0 \times 10^{-5}$ gauss the spectral index is -2.17 when $T=10$.

\section{Conclusions}

The relativistic MB distribution has been derived in [10] without any particular statistics: here we derived, when the main variable is the Lorentz factor $\gamma$, the 
constant of normalization, the average value, the second moment about the origin, the variance, the mode, the asymptotic behavior, an approximate expression for the average value as function of the temperature and an inverted expression for the temperature as function of average value.

We derived the following statistical parameters of the MJ distribution when $\gamma$ is the main variable: average value, variance, mode, asymptotic expansion, two approximate expressions for the distribution function, a first evaluation of $\Theta$ from the average value and a second evaluation of $\Theta$ from the variance.

Following the usual argument which suggests a power law behavior for the spectral distribution of the synchrotron emission in presence of a power law distribution for the energy of the electrons, we derived the spectral distribution for the relativistic $\mathrm{MB}$ and $\mathrm{MJ}$ distributions which are now function of the selected generalized temperature and the magnetic field. Two astrophysical applications are given: the spectral distribution of emission in the core of M87 in the framework of the synchrotron emissivity and an explanation for the steep spectra sources in the framework of the synchrotron emissivity for the relativistic MJ distribution.

\section{Conflicts of Interest}

The author declares no conflicts of interest regarding the publication of this paper.

\section{References}

[1] Maxwell, J.C. (1860) V. Illustrations of the Dynamical Theory of Gases.-Part I. On the Motions and Collisions of Perfectly Elastic Spheres. The London, Edinburgh, and Dublin Philosophical Magazine and Journal of Science, 19, 19-32. https://doi.org/10.1080/14786446008642818

[2] Boltzmann, L. (1872) Weitere Studien über das Wärmegleichgewicht unter Gasmolekülen. Sitzungsberichte Akademie der Wissenschaften, 66, 275-370.

[3] Jüttner, F. (1911) Das maxwellsche gesetz der geschwindigkeitsverteilung in der relativtheorie. Annalen der Physik, 339, 856. https://doi.org/10.1002/andp.19113390503

[4] Synge, J. (1957) The Relativistic Gas. North-Holland, New York.

[5] Livadiotis, G. (2016) Modeling Anisotropic Maxwell-Jüttner Distributions: Derivation and Properties. Annales Geophysicae, 34, 1145-1158. https://doi.org/10.5194/angeo-34-1145-2016

[6] Tsouros, A. and Kylafis, N.D. (2017) The Energy Distribution of Electrons in Radio Jets. $A \& A, 603$, L4. https://doi.org/10.1051/0004-6361/201730749

[7] Aragón-Muñoz, L. and Chacón-Acosta, G. (2018) Modified Relativistic Jüttner-Like Distribution Functions with $\eta$-Parameter. Journal of Physics. Conference Series, 1030, Article ID: 012004. https://doi.org/10.1088/1742-6596/1030/1/012004

[8] Sadegzadeh, S. and Mousavi, A. (2018) Maxwell-Jüttner Distributed Counterstreaming Magnetoplasmas-Parallel Propagation. Physics of Plasmas, 25, Article ID: 112107. https://doi.org/10.1063/1.5054830

[9] Freund, J. (2008) Special Relativity for Beginners: A Textbook for Undergraduates. 
World Scientific Press, Singapore. https://doi.org/10.1142/6601

[10] Claycomb, J. (2018) Mathematical Methods for Physics: Using MATLAB and Maple. Mercury Learning \& Information, Boston.

[11] Meijer, C. (1936) Über Whittakersche bzw. Besselsche Funktionen und deren Produkte. Nieuw Archief voor Wiskunde, 18, 10-39.

[12] Meijer, C. (1941) Multiplikationstheoreme fur die Funktion $G_{p, q}^{m, n}(z)$. Proceedings of the Koninklijke Nederlandse Akademie, 44, 1062-1070.

[13] Olver, F.W.J., Lozier, D.W., Boisvert, R.F. and Clark, C.W. (2010) NIST Handbook of Mathematical Functions. Cambridge University Press, Cambridge.

[14] Brandt, S. and Gowan, G. (1998) Data Analysis: Statistical and Computational Methods for Scientists and Engineers. Springer \& Verlag, New York.

[15] Press, W.H., Teukolsky, S.A., Vetterling, W.T. and Flannery, B.P. (1992) Numerical Recipes in FORTRAN. The Art of Scientific Computing. Cambridge University Press, Cambridge.

[16] Hagedorn, T.R. (2000) General Formulas for Solving Solvable Sextic Equations. Journal of Algebra, 233, 704. https://doi.org/10.1006/jabr.2000.8428

[17] Anton, H., Bivens, I. and Davis, S. (2012) Calculus. 10th Edition, Wiley, New York.

[18] Longair, M.S. (2011) High Energy Astrophysics. 3rd Edition, Cambridge University Press, Cambridge.

[19] Abramowitz, M. and Stegun, I.A. (1965) Handbook of Mathematical Functions with Formulas, Graphs, and Mathematical Tables. Dover, New York. https://doi.org/10.1063/1.3047921

[20] Oldham, K.B., Myland, J. and Spanier, J. (2010) An Atlas of Functions: With Equator, the Atlas Function Calculator. Springer Science \& Business Media, New York. https://doi.org/10.1007/978-0-387-48807-3

[21] von Seggern, D. (1992) CRC Standard Curves and Surfaces. CRC, New York.

[22] Thompson, W.J. (1997) Atlas for Computing Mathematical Functions. WileyInterscience, New York.

[23] Prieto, M.A., Fernández-Ontiveros, J.A., Markoff, S., Espada, D. and GonzálezMartn, O. (2016) The Central Parsecs of M87: Jet Emission and an Elusive Accretion Disc. MNRAS, 457, 3801. https://doi.org/10.1093/mnras/stw166

[24] De Breuck, C., van Breugel, W., Röttgering, H.J.A. and Miley, G. (2000) A Sample of 669 Ultra Steep Spectrum Radio Sources to Find High Redshift Radio Galaxies. Astronomy and Astrophysics Supplement Series, 143, 303.

https://doi.org/10.1051/aas:2000181 